\title{
Semi-Analytic Model for Dispersion Relation of Nanowire Lasers
}

\author{
Mohammad-Azim Karami ${ }^{1}$, Mehdi Ahmadi-Boroujeni ${ }^{2}$, Ali Afzali-Kusha ${ }^{1}$, and Reza Faraji-Dana ${ }^{2}$ \\ ${ }^{1}$ Nanoelectronics Center of Excellence ${ }^{2}$ Center of Excellence for Applied Electromagnetics Systems \\ School of Electrical and Computer Engineering University of Tehran, Iran, \\ m.a.karami@ece.ut.ac.ir, ahmadi.b@ece.ut.ac.ir, afzali@ut.ac.ir, and reza@ut.ac.ir
}

\begin{abstract}
In this paper, a semi-analytical model for the dispersion relation of nanowire lasers is presented. The approach which is based on the multi-conductor transmission line modeling, makes use of the Fourier expansion technique. The cross-section of the nanowire is assumed to be hexagonally-shaped. To determine the accuracy, the model results are compared to the numerical results obtained from FDTD (finite difference time domain) technique. The model error when using 40 layers in the multi-conductor transmission line modeling and keeping 8 Fourier expansion elements is about 13 percent. The error is a function of the number of layers, the distance between the set of nanowires assumed for Fourier expansion, and the number of Fourier elements considered in the model.
\end{abstract}

\section{Keywords}

Nanowire, Dispersion Relation, Transmission Line

\section{INTRODUCTION}

Semiconductor nanowires are one-dimensional nanostructures emerging as promising building blocks for nanoscale photonic devices and circuits [1]. They have been utilized as key elements in single and multicolor nanoscale light-emitting diodes, polarization sensitive photodetectors, and optically and electrically driven lasers, in which single nanowires both define the optical cavity and are the active lasing medium [1]. The interest in optical properties of semiconductor nanowires is because of the cylindrical geometry and strong two-dimensional confinement of electrons, holes and photons which make them particularly attractive as potential building blocks for nanoscale electronics and optoelectronic devices, including lasers and nonlinear optical frequency converters [2].

A remarkable feature of nanowires is the ability to support guided modes and to provide gain to the modes. This dual functionality leads to a significant reduction of the laser size and simplification in fabrication [3]. Near-field images of lasing nanowires show emission through their end facets. This lasing can be attributed to the guided modes propagating along the axial direction. While nanowires with small radii can support only the $\mathrm{HE}_{11}$ mode, nanowires with larger radii can support several other modes, in particular, $\mathrm{TM}_{01}$ and $\mathrm{TE}_{01}[4]$.

By a proper control of the growth process, doping, and other material parameters, unique electronic and optical properties could be created for nanowires which have typical dimension of 20-400 $\mathrm{nm}$ in diameter and 2-40 of microns in length [5].
Nanowires are grown in a $2 \mathrm{D}$ array vertical to the substrate providing an interesting example of vertical cavity surface emitting lasers array. Recently, lasing was demonstrated in $\mathrm{ZnO}$, $\mathrm{GaN}$, and $\mathrm{CdS}$ nanowires [6]. The relative dielectric constants of $\mathrm{GaN}, \mathrm{ZnO}$, and $\mathrm{CdS}$ are considered to be 6 at the lasing frequencies [7].

In this paper, a semi-analytical model for the dispersion relation derivation of nanowire lasers is presented. Section 2 reviews the previous works related to the modeling of the nanowire dispersion relation while the multi-conductor transmission line model for the dispersion relation derivation of nanowire lasers is discussed in Section 3. The results are discussed in Section 4 with the summary and conclusions are given in Section 5.

\section{REVIEW OF PREVIOUS WORKS}

In general, the end facets of a nanowire can serve as the two mirrors of a Fabry-Perot etalon which acts as a cavity when photons propagate along the axial direction of the nanowire [5]. A remarkable feature of the nanowire is the ability to support guided modes and provide gain to them [3]. Because of their small lateral dimension and strong optical confinement, nanowires usually show complex dispersion relations [5].

Different optical mode properties and end facet refelectivities of the nanowire laser structures have been modeled [5][7]. In [7], nanowires are considered as cylindrical shapes whose dispersion properties may be modeled using a full analytical approach. It uses the simplified assumption that the nanowires are rotationally symmetric nano-cylinders, regardless of their different crosssectional shapes. The simplification reduces a full 3-dimensional problem to a 2-dimensional one considerably reducing the computational overhead of the modeling [5]. Since all GaN and $\mathrm{ZnO}$ nanowire structures reported have hexagonally shaped cross sections, the model presented in [7] may not be accurate enough for these structures [5]. This geometry has been resulted due to the Wurzite crystalline structure of these materials.

In [5], the dispersion properties of the nanowires with the hexagonally shaped cross-section have been studied using 3dimensional FDTD (Finite Difference Time Domain) simulations. In the FDTD calculations, an initial Gaussian shaped field profile is used to excite the electromagnetic wave traveling along the $z$ axis of the nanowire. Then, the frequency domain components are recorded to obtain the corresponding dispersion relation (the relation between $\beta$, the propagation constant of a propagating mode, at every given angular frequency $\omega_{0}$ ). 
Although a large number of numerical methods like FDTD are applicable for analyzing optical waveguiding structures, they usually suffer from several limitations imposed by discretization of the solution space or the required absorbing boundary conditions [8]. On the other hand, approximate analytical methods are not satisfactory and versatile, because most of them are scalar methods that can only analyze waveguides having diagonal index tensors [8]. In next section, we describe a semi-analytical approach for the dispersion relation of the nanowires.

\section{MULTI-CONDUCTOR TRANSMISSION LINE MODEL FOR NANOWIRES}

In this method, as a first step, the nanowire is periodically repeated in one transverse coordinate direction with an appropriate period $L_{x}$, as shown in Figure 1. The resulting periodic structure can be analyzed using a systematic matrix approach. By making the period asymptotically large, the dispersion relation of the periodic structure approaches those of the original nanowire [9].

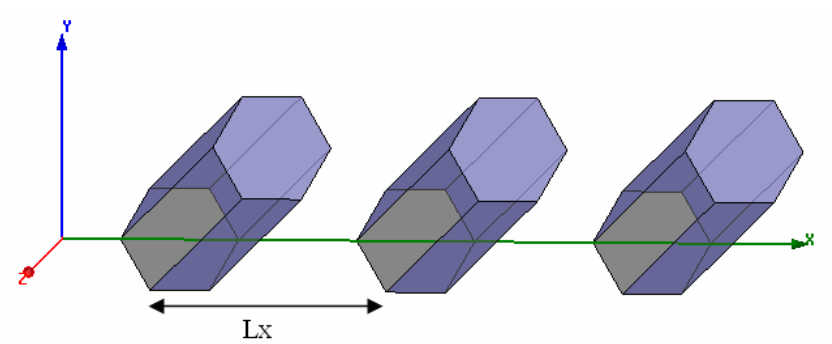

Figure 1. Nanowire repeated with period $L_{x}$ along the $x$-axis.

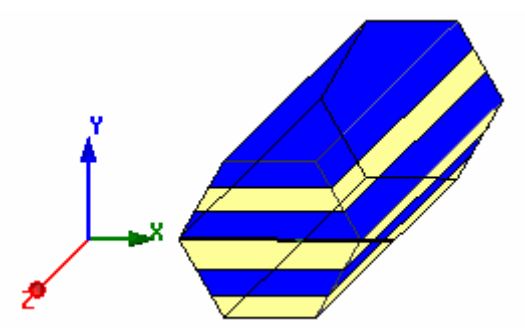

Figure 2. Considering different layers for cross section of nanowire.

This method which is also called plane-wave expansion technique is categorized as a Fourier expansion technique and has been largely used for analyzing periodic structures such as dielectric gratings and photonic crystals [9]. In conjunction with the Fourier expansion techniques, a transmission line (TL) network model may be used for analyzing these structures with greater efficiency and physical insight [9]. Now, we use the technique for the nanowires.

The cross section of the nanowire is divided into different layers where the TL characteristics are considered for each layer as shown in Figure 2. The voltage and current variables of the applied generalized TL model are chosen to be the tangential components of the electric and magnetic fields such that the technique provides a unified formulation. The formulation satisfies both the boundary and the power conservation conditions in all regions of the nanowire problem.

Assuming the wave propagation along the $z$-axis, and after some algebraic manipulations of the Maxwell's curl equations in the $n$th layer of the waveguide, one obtains [9]

$$
\frac{d}{d y}\left(\begin{array}{c}
{\left[E_{z}\right]} \\
{\left[E_{x}\right]} \\
{\left[H_{x}\right]} \\
-\left[H_{z}\right]
\end{array}\right)=\left(\begin{array}{cc}
{[A]} & -j \omega[L] \\
-j \omega[C] & {[B]}
\end{array}\right)\left(\begin{array}{c}
{\left[E_{z}\right]} \\
{\left[E_{x}\right]} \\
{\left[H_{x}\right]} \\
-\left[H_{z}\right]
\end{array}\right)
$$

where the matrices $[\mathrm{A}],[\mathrm{B}],[\mathrm{L}]$ and $[\mathrm{C}]$ are of $2 \mathrm{~K} \times 2 \mathrm{~K}$ dimension, and are given by

$$
\begin{gathered}
{[A]=\left(\begin{array}{cc}
j \gamma\left[N_{y y}^{2}\right]^{-1}\left[N_{y z}^{2}\right] & j \gamma\left[N_{y y}^{2}\right]^{-1}\left[N_{y x}^{2}\right] \\
j[\alpha]\left[N_{y y}^{2}\right]^{-1}\left[N_{y z}^{2}\right] & j[\alpha]\left[N_{y y}^{2}\right]^{-1}\left[N_{y x}^{2}\right]
\end{array}\right)} \\
{[L]=\mu_{0}\left(\begin{array}{cc}
1_{K \times K}-\bar{\gamma}^{2}\left[N_{y y}^{2}\right]^{-1} & -\bar{\gamma}\left[N_{y y}^{2}\right]^{-1}[\bar{\alpha}] \\
-[\bar{\alpha}]\left[N_{y y}^{2}\right]^{-1} \bar{\gamma} & 1_{K \times K}-[\bar{\alpha}]\left[N_{y y}^{2}\right]^{-1}[\bar{\alpha}]
\end{array}\right)} \\
{[C]=\varepsilon_{0}\left(\begin{array}{c}
{\left[N_{z z}^{2}\right]-[\bar{\alpha}]^{2}-\left[N_{z y}^{2}\right]\left[N_{y y}^{2}\right]^{-1}\left[N_{y z}^{2}\right]} \\
{\left[N_{x z}^{2}\right]+\bar{\gamma}[\bar{\alpha}]-\left[N_{x y}^{2}\right]\left[N_{y y}^{2}\right]^{-1}\left[N_{y z}^{2}\right]} \\
{\left[N_{x z}^{2}\right]+\bar{\gamma}[\bar{\alpha}]-\left[N_{x y}^{2}\right]\left[N_{y y}^{2}\right]^{-1}\left[N_{y z}^{2}\right]} \\
{\left[N_{x x}^{2}\right]-\bar{\gamma}^{2} 1_{K \times K}-\left[N_{x y}^{2}\right]\left[N_{y y}^{2}\right]^{-1}\left[N_{y x}^{2}\right]}
\end{array}\right)}
\end{gathered}
$$

and

$$
[B]=\left(\begin{array}{ll}
j \gamma\left[N_{z y}^{2}\right]\left[N_{y y}^{2}\right]^{-1} & {\left[N_{z y}^{2}\right]\left[N_{y y}^{2}\right]^{-1}[\alpha]} \\
j \gamma\left[N_{x y}^{2}\right]\left[N_{y y}^{2}\right]^{-1} & {\left[N_{x y}^{2}\right]\left[N_{y y}^{2}\right]^{-1}[\alpha]}
\end{array}\right)
$$

Here, $k_{0}=\omega \sqrt{\mu_{0} \varepsilon_{0}},[\bar{\alpha}]=[\alpha] / k_{0}, \bar{\gamma}=\gamma / k_{0}$, and

$\left[N_{u v}^{2}\right]=\left[\begin{array}{cccc}\varepsilon_{u v, 0} & \varepsilon_{u v,-1} & \cdots & \varepsilon_{u v,-2 M} \\ \varepsilon_{u v, 1} & \varepsilon_{u v, 0} & \cdots & \varepsilon_{u v,-2 M+1} \\ \vdots & \vdots & \ddots & \vdots \\ \varepsilon_{u v, 2 M} & \varepsilon_{u v, 2 M-1} & \cdots & \varepsilon_{u v, 0}\end{array}\right]$

where $u, v \in\{x, y, z\}$ and $\varepsilon_{u v, m}=\frac{1}{L_{x}} \int_{-\frac{L_{x}}{2}}^{\frac{L_{x}}{2}} \varepsilon_{u v}(x, y) e^{j \frac{2 m \pi}{L_{x}} x} d x$.

To decouple the system of equations in (1), the voltages $[\mathrm{V}(\mathrm{y})]=\left(\left[\mathrm{E}_{\mathrm{z}}\right],\left[\mathrm{E}_{\mathrm{x}}\right]\right)^{\mathrm{T}}$ and the currents $[\mathrm{I}(\mathrm{y})]=\left(\left[\mathrm{H}_{\mathrm{x}}\right],-\left[\mathrm{H}_{\mathrm{z}}\right]\right)^{\mathrm{T}}$ are considered on a multi-conductor transmission line [8]. The total voltage and current on the transmission line of the layer under consideration can be expressed in terms of the eigensolutions as [9]:

$$
\left(\begin{array}{l}
{[V(y)]} \\
{[I(y)]}
\end{array}\right)=\left(\begin{array}{l}
{[P]} \\
{[Q]}
\end{array}\right)\left[e^{-j k\left(y-y_{n}\right)}\right][a]+\left(\begin{array}{c}
{[\widetilde{P}]} \\
-[\widetilde{Q}]
\end{array}\right)\left[e^{j \tilde{k}\left(y-y_{n-1}\right)}\right][b]
$$

where $[a]$ and $[b]$ are constant $2 K \times 1$ matrices whose entries are $a_{i}$ and $b_{\mathrm{i}} \cdot\left[\mathrm{e}^{-\mathrm{j} k(y-y)}{ }_{n}\right]$ and $\left.\left[\mathrm{e}^{\mathrm{j} k(y-y}{ }_{n-1}\right)^{\prime}\right]$ are $2 K \times 2 K$ dimensional 
diagonal matrices. The diagonal elements of the matrices are $\mathrm{e}^{-\mathrm{j} k i}$ ${ }^{(y-y)}$ and $\left.\mathrm{e}^{\mathrm{j} k i(y-y}{ }_{n-1}\right)$ for $i=1$ to $2 K$. Also, in (4), $[P],[Q],[\widetilde{P}]$, and [ $\widetilde{Q}]$ are $2 K \times 2 K$ matrices composed of $\left[p_{i}\right],\left[q_{i}\right],\left[\tilde{p}_{i}\right]$, and $\left[\widetilde{q}_{i}\right]$ as their columns, respectively. The eigenvalue $\left(k_{m}\right)$ and the corresponding eigenvectors $\left(\left[\mathrm{p}_{\mathrm{m}}\right],\left[\mathrm{q}_{\mathrm{m}}\right]\right)^{\mathrm{T}}$ is obtained from solving the eigenvalue problem given by

$$
\left(\begin{array}{cc}
j[A] & -j \omega[L] \\
-j \omega[c] & {[B]}
\end{array}\right)\left(\begin{array}{l}
{[p]} \\
{[q]}
\end{array}\right)=-j k\left(\begin{array}{l}
{[p]} \\
{[q]}
\end{array}\right)
$$

The eigensolutions are arranged into two sets. One set of the eigenvalues represents waves traveling in the $+y$ direction and the other set contains the eigenvalues for the wave traveling in the $-y$ direction. The propagation constants and the eigenvectors of these sets are denoted by $\left\{k_{i}\right.$ and $\left.\left(\left[p_{i}\right],\left[q_{i}\right]\right)^{T}\right\}$ and $\left\{-\tilde{k}_{i}\right.$ and $\left(\left[\tilde{p}_{i}\right]\right.$, $\left.\left.-\left[\widetilde{q}_{i}\right]\right)^{T}\right\}$, respectively, for $i=1$ to $2 K$.

As Equation (4) suggests, the matrices $[P],[Q],[\widetilde{P}]$, and $[\widetilde{Q}]$ transform the coupled voltages and currents on the multiconductor TL $([V(y)]$ and $[I(y)])$ into a set of decoupled traveling waves denoted by $[a(y)]$ and $[b(y)]$. These waves are given by

$$
\begin{aligned}
& {[a(y)]=\left[e^{-j k\left(y-y_{n}\right)}\right][a]} \\
& {[b(y)]=\left[e^{-j \tilde{k}\left(y-y_{n}\right)}\right][b]}
\end{aligned}
$$

The formulations presented up to this point leads to a generalized TL model for each layer of an optical waveguide. This model consists of a generalized TL which represents the wave propagation along the $y$-direction in that layer and four transformation matrices that are used to connect the TL of the layer to that of its upper and lower layers as shown in Figure 3.

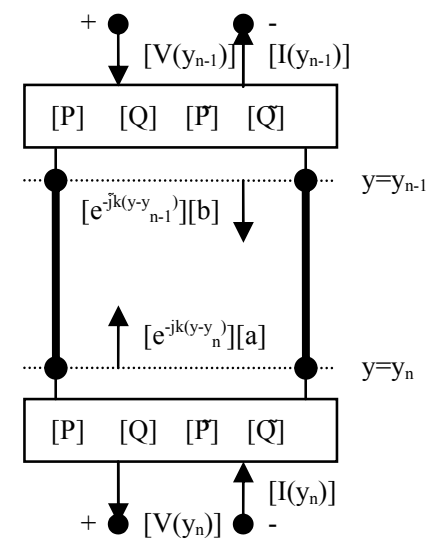

Figure 3. TL model for the $n$th layer of a waveguide [8].

Using the model in Figure 3, one can construct a network model for the nanowire by interconnecting the TL model of its individual layers. At the interfaces of the layers, the continuity of the tangential electric and magnetic fields, i.e., $E_{x}, E_{z}, H_{x}$, and $H_{z}$, must be satisfied. It can be shown that the continuity of tangential field components is equivalent to the continuity of their respective single-column matrices, i.e. $\left[E_{x}\right],\left[E_{z}\right],\left[H_{x}\right]$, and $\left[H_{z}\right]$, which also corresponds to the continuity of line voltages and currents [10].

Consider the voltages and currents on these transmission lines at $y$ $=\mathrm{y}_{0}$ where $\mathrm{y}_{0}$ can take an arbitrary value. At $y=y_{0}$, let $[a]$ and $[b]$ be the amplitudes of incident and reflected waves, respectively. These amplitudes are related to each other through

$$
[b]=\left[\Gamma_{y \geq y_{0}}\right][a]
$$

Here, $\left[\Gamma_{y \geq y 0}\right]$ is the reflection coefficient matrix looking upward into the multi-conductor transmission line. Now, if the wave propagation for $y \leq y_{0}$ is considered, the reflected wave in the last case plays the role of the incident wave and vice versa. Hence,

$[a]=\left[\Gamma_{y \leq y_{0}}\right][b]$

Where $\left[\Gamma_{y \leq y 0}\right]$ is the reflection coefficient matrix at $y=y_{0}$ looking downward. Eliminating $[b]$ from (7) and (8) leads to

$\left(\left[\Gamma_{y \leq y_{0}}\right]\left[\Gamma_{y \geq y_{0}}\right]-1_{2 K \times 2 K}\right)[a]=0_{2 K \times 1}$

Thus, to have non-trivial solutions for $[a]$, we should have

$\operatorname{det}\left(\left[\Gamma_{y \leq y_{0}}\right]\left[\Gamma_{y \geq y_{0}}\right]-1_{2 K \times 2 K}\right)=0$

Now, the above equation is to be solved for the only unknown $\beta$, the propagation constant of a propagating mode, at every given angular frequency $\omega_{0}$. Having these propagation constants, the corresponding eigenvectors $[a]$ and $[b]$ may be found from (9) and (7), respectively. Finally, the transformed voltages and currents at any given value of $y$ may be found [8].

\section{RESULTS AND DISCUSSION}

A hexagonal shaped nanowire is considered for the dispersion relation derivation with the diameter of $80 \mathrm{~nm}$ and the length of $3 \mu \mathrm{m}$. The relative dielectric constant, $\varepsilon_{r}$, is considered to be 6.5 which is close to a GaN nanowire lasers. First, the cross section of the nanowire is divided into 9 layers and only 8 Fourier elements are considered for limiting the simulation time of the semianalytic model. The separation between each two adjacent nanowires, $L_{x}$, is considered to be $120 \mathrm{~nm}$. To assess the accuracy of the model, we have used the finite difference time domain (FDTD) numerical simulations. Figure 4 shows the dispersion relation results obtained by the TL model and the FDTD simulator used in [5]. The FDTD results are shown by solid line and the semi-analytic model results are shown by circles. An average error of 26 percent between the dispersion relation calculated by the semi-analytic model proposed in this work and the FDTD results are obtained.

The accuracy is expected to increase when the number of layers is increased. As the next case, the cross section of the nanowire is divided to 20 layers while keeping the 8 Fourier elements as before. Also, $L_{x}$ is again considered to be $120 \mathrm{~nm}$. Figure 5 shows the comparison between the results of the model and those of the FDTD simulator. In this case the average error has been reduced to 22 percent. 


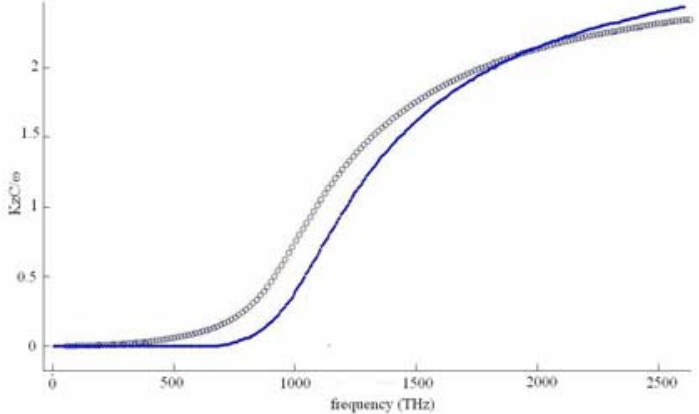

Figure 4. Dispersion relation of nanowire by considering 9 different layers.

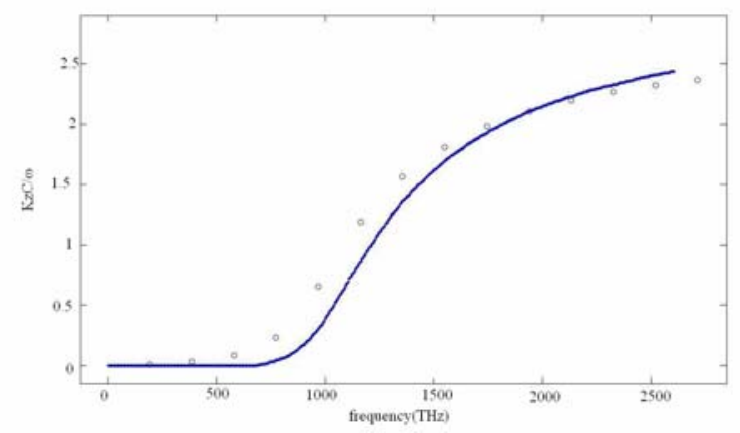

Figure 5. Dispersion relation of nanowire by considering 20 different layers.

Figure 6 shows the error of 18 percent for the case of considering 15 Fourier elements and 20 different layers for the nanowire in compare with FDTD results.

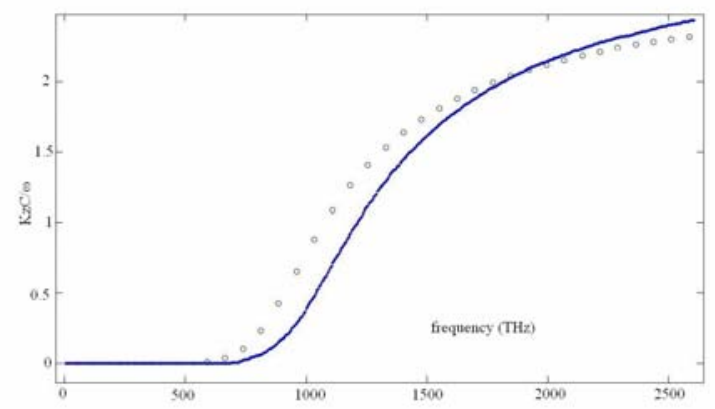

Figure 6. Dispersion relation of nanowire by considering 20 different layers for it with 15 Fourier elements.

Finally, the dispersion relation of nanowire is compared by considering 40 different layers for the nanowire and 8 Fourier elements for limiting the simulation time of the semi-analytic model, as shown in figure 7. An error factor of 13 percent is convenient for the dispersion relation calculated by semi-analytic method in compare with the time consuming FDTD computational method as shown in this figure.

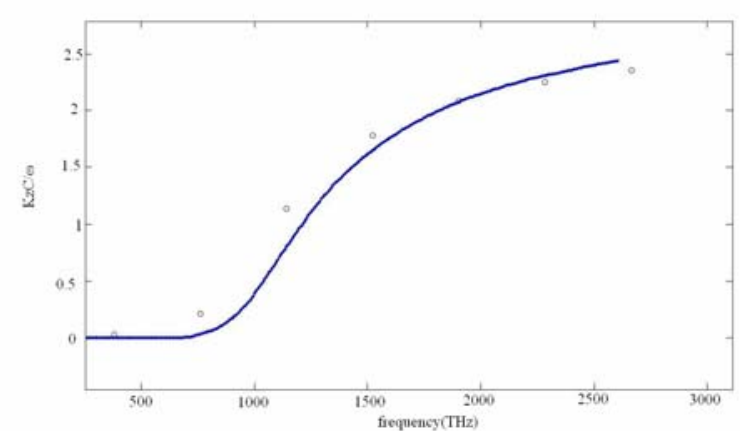

Figure 7. Dispersion relation of nanowire by considering 40 different layers.

\section{SUMMARY AND CONCLUSION}

In this paper, a semi-analytical model for the dispersion relation of the nanowire based on the Fourier expansion technique and the transmission line modeling was presented. The dispersion relation obtained using this approach was compared to those of the finite difference time domain (FDTD) numerical method to assess the error of the proposed technique. The errors were calculated for different number of layers and different number of considered Fourier elements. The error was as low as 13 percent when considering 40 layers for the cross-section of the nanowire with 8 Fourier elements. This error will be reduced by increasing the number of layers of the nanowire in the transmission line modeling, increasing the distance between the set of nanowires considered for the Fourier expansion, and increasing the number of Fourier elements included in the simulation. Finally, note that the use of the semi-analytical modeling of the nanowire dispersion relation could provide physical insights which can be used for a better understanding of the optical properties of the device with less simulation time if it is incorporated in a simulator.

\section{REFERENCES}

[1] A .B . Greytak, C. J. Barrelet, Y . Li, C .M .Lieber, "Semiconductor nanowire laser and nanowire waveguide electro-optic modulators" Applied Physics Letters, 151103 2005, Lett 87.

[2] J .C. Johnson, H .J . Choi, K. P. Knutsen, R. D .Schaller, P. Yang, and R .J . Saykally, "Single gallium nitride nanowire lasers “,Nature, Vol. 1. October 2002, pp 106-110.

[3] A. V. Maslov, and C. Z. Ning," Modal Gain in a Semiconductor Nanowire Laser With Anisotropic Bandstructure " IEEE Journal of Quantum Electronics, Vol. 40, No. 10, October 2004, pp.1389-1397.

[4] A. V. Maslov, and C. Z. Ning," Far-field emission of a semiconductor nanowire laser ", Optics letters, Vol. 29, No. 6, March 2004, pp. 572-574.

[5] L. Chen," Modeling and simulation of optically pumped micro-cavity semiconductor laser structures", Ph.D. Thesis, Carnegie Mellon University, 2005.

[6] S. Gradecak, F. Qian, Y . Li, mH. Park, and C. M. Lieber, "GaN nanowire lasers with low lasing thresholds", Applied Physics Letters, 87, 173111, October 2005. 
[7] A. V. Maslov, and C. Z. Ning,"'Reflection of guided modes in a semiconductor nanowire laser", Applied Physics letters, Vol. 83,No. 6, August 2003, pp.1237-1239.

[8] M. A. Boroujeni, and M. Shahabadi," Modal analysis of multilayer planar lossy anisotropic optical waveguides", Journal of optics: pure and applied optics, 2006, pp-856863.
[9] M. A. Boroujeni, and M. Shahabadi," Full-wave analysis of lossy anisotropic optical waveguides using a transmission line approach based on a Fourier method ", Journal of optics: pure and applied optics, 2006, pp. 1080-1087. 